\title{
Retrograde Maculopathy in Patients With Glaucoma
}

\author{
Jacqueline Brazerol, BSc,* Milko E. Iliev, MD,* René Höhn, MD, *† \\ Stephan Fränkl, MD,* Hilary Grabe, MD,* and Mathias Abegg, MD, PhD*
}

\begin{abstract}
Purpose: Macular optical coherence tomography (OCT) analysis can be used for quantitative measures of optic nerve atrophy at a location far from the optic nerve head. This recently led to the finding of microcystic macular edema (MME), that is vacuolar inclusions in the macular inner nuclear layer, in some glaucoma patients. The involvement of individual retinal layers is yet unclear in glaucoma. In this study we systematically investigated glaucoma-induced changes in macular layers to evaluate whether glaucoma-associated damage extends beyond the macular ganglion cell layer.
\end{abstract}

Patients and Methods: We included 218 consecutive patients and 282 eyes with confirmed primary open-angle glaucoma or pseudoexfoliation glaucoma, and macular OCT in a cross-sectional observational study. Eyes were screened for presence of MME. Thickness of individual retinal layers was determined using a semiautomatic segmentation algorithm. Peripapillary nerve fiber layer thickness and mean defect in visual field testing were extracted from OCT and medical records, respectively. Results were compared with a small group of eyes with no apparent glaucoma.

Results: We found MME in 5 eyes from 5 primary open-angle glaucoma patients and 3 eyes of 3 pseudoexfoliation glaucoma patients $(2.8 \%)$. MME was confined to the inner nuclear layer in a perifoveal ring and was associated with thinning of the ganglion cell layer and thickening of the macular inner nuclear layer. Glaucoma eyes without MME showed a significant inverse correlation of inner nuclear layer thickness with glaucoma severity.

Conclusions: Glaucomatous damage leads to a gradual thickening of the inner nuclear layer, which leads to MME in more severe glaucoma cases. These changes, along with nerve fiber loss and ganglion cell loss, may be summarized as glaucoma-associated retrograde maculopathy.

Key Words: retina, maculopathy, glaucoma, segmentation, macular edema

( J Glaucoma 2017;26:423-429)

A reliable determination of glaucoma severity and progression rate is key to the successful management of this common disease, which imposes a considerable burden of disease both on affected patients and society.

Received for publication June 29, 2016; accepted December 30, 2016. From the *Department of Ophthalmology, Inselspital, Bern University Hospital, University of Bern, Bern, Switzerland; and $†$ Department of Ophthalmology, University Medical Center Mainz, Mainz, Germany.

Disclosure: The authors declare no conflict of interest.

Reprints: Mathias Abegg, MD, PhD, Department of Ophthalmology, Bern University Hospital, University of Bern, Inselspital, CH-3010 Bern, Switzerland (e-mail: mathias.abegg@insel.ch).

Copyright (c) 2017 Wolters Kluwer Health, Inc. All rights reserved.

DOI: $10.1097 /$ IJG.0000000000000633
Traditionally, the glaucoma-associated nerve fiber loss is assessed at the level of the optic disc, where it causes increased disc cupping and thinning of the retinal nerve fiber layer (RNFL). The latter may be measured using spectral-domain optical coherence tomography, which provides reproducible imaging at high spatial resolution. RNFL measurements have brought great advances in objective quantitative assessment of glaucoma progression ${ }^{1,2}$ outperforming every other imaging device so far. ${ }^{3}$ Given that all nerve fibers pass through the optic nerve head, it is logical to measure fiber loss at this level. However, there are numerous situations in which RNFL measures are difficult or impossible to use: advanced glaucomatous disc damage, optic disc drusen, concurrent intracranial hypertension, dysplastic discs, myopic disc alterations, etc. Thus a measure of glaucoma-associated retinal nerve fiber loss distant to the optic disc is useful and has inspired research in the recent past. Most research has focused, with good reason, on the analysis of the macular ganglion cell layer (mGCL) complex representing the area with the highest density of retinal ganglion cells in the retina (about 50\%). ${ }^{4}$ Studies found that the $\mathrm{mGCL}$ and inner plexiform layer (IPL), the macular ganglion cell complex $(\mathrm{mGCC}=\mathrm{RNFL}+\mathrm{GCL}+\mathrm{IPL})$, and the total macular thickness can be used to detect glaucoma progression. ${ }^{5,6}$ Less explored is the effect of glaucoma on deeper retinal layers. It was found that glaucoma might be associated with changes at the level of photoreceptors. ${ }^{7,8}$ The involvement of the inner nuclear layer is not clear: Some have reported that outer retinal layer thickness and inner nuclear layer thickness is not changed from glaucoma $^{5,9}$ while others have found a thinning of the inner nuclear layer. ${ }^{10}$ Recent works from nonglaucomaassociated optic neuropathies showed that optic neuropathy may lead to thickening and edema in the inner nuclear layer, which was termed microcystic macular edema (MME). ${ }^{11}$ Nerve fiber damage and the subsequent retrograde loss of ganglion cells is thought to cause a dysfunction of Muller cells, which impairs retinal water clearance and results in edema. ${ }^{12-15}$ Muller cell gliosis is indeed a common finding in animal models of optic neuropathy ${ }^{16,17}$ and in humans. ${ }^{18} \mathrm{MME}$ has been found in all types of optic neuropathy including glaucoma, ${ }^{19}$ with a reported prevalence of $4 \%$ in the latter and $9 \%$ in nonglaucoma optic neuropathies. ${ }^{13}$ It is unclear, however, why some but not other patients develop this edema. On the basis of the Muller cell dysfunction hypothesis one would predict a thickness change, with or without microcysts, in most cases with pronounced optic neuropathy. Given the potential of macular layer analysis for future assessment of glaucoma damage and the observation of MME in glaucoma, we believe that the analysis of the macular anatomy, inner and outer layers, is important in glaucoma. 
In this work we systematically explored individual retinal layers in the macula of patients with primary openangle glaucoma (POAG) and pseudoexfoliation glaucoma (PEXG) to determine thickness in and below the GCL and to correlate these parameters with the severity of glaucoma. We determined the frequency of MME in POAG and PEXG and explored changes in the deeper layers associated with glaucoma damage.

\section{PATIENTS AND METHODS}

\section{Study Design and Patients}

All medical records of patients seen between January 2010 and January 2015 at the Department of Ophthalmology, Inselspital, University Hospital, University of Bern, were retrospectively screened for the German translation of the keywords "open-angle glaucoma" (POAG) and "pseudoexfoliation glaucoma" (PEXG). Inclusion criteria were (1) confirmed diagnosis of either POAG or PEXG by a glaucoma specialist and (2) presence of high resolution macular OCT. The diagnosis of glaucoma was based on standard clinical criteria, that is presence of optic neuropathy, elevated intraocular pressure in the present or the past, and visual field defects. Glaucoma suspects or normal tension glaucoma were thus not included. Exclusion criteria were the following: Known history of retinal disease (eg, diabetic retinopathy, age-related macular degeneration, retinal detachment, etc.), optic nerve abnormalities other than glaucoma, and poor image quality. The study was approved by the local ethics committee.

\section{Demographics and Clinical Characteristics}

For each patient we determined age and sex. If available we also collected the best-corrected visual acuity, intraocular pressure on the last visit, and mean defect (MD) in visual field determined by the "glaucoma program" of the Octopus Perimeter (Haag-Streit, Switzerland). Visual acuity was converted from Snellen values into logMAR values according to a previously published conversion table $^{20}$ to allow averaging and statistical analysis.

\section{Imaging, Segmentation, and Analysis}

For imaging, a high-resolution OCT with a scanning laser ophthalmoscope (Spectralis HRA + OCT; Heidelberg Engineering, Heidelberg, Germany) was used. This allows for simultaneous OCT scans with infrared imaging. The OCT scans included a volume scan $(30 \times 20$ degrees $)$ using
61 line scans, each consisting of 9 averaged line scans. Macular line scans were screened manually for presence of MME which was defined by the presence of vertical vacuoles in the inner nuclear layer.

For the analysis of retinal layers we used a custombuilt segmentation software as described previously. ${ }^{13,21}$ The segmentation algorithm determined the border between (1) the internal limiting membrane; (2) GCL; (3) inner nuclear layer; (4) outer nuclear layer; (5) junction of the inner and the outer segments; (6) outer segment of photoreceptors/pigment epithelium complex; and (7) Bruch membrane. From this we determined the thickness of the combined $\mathrm{mGCL}$ and the IPL, macular inner nuclear layer thickness and outer plexiform layer (mINL/OPL) and macular outer retinal thickness (mORT). The latter was defined as the thickness from the inner border of the outer nuclear layer down to Bruch membrane. The automatic segmentation was verified by an examiner (J.B.) and, when necessary, manually adapted. To obtain layer thickness values an "Early Treatment Diabetic Retinopathy Study (ETDRS)"- grid was centered on the fovea and the average thickness value for each sector of the grid was exported. To simplify the further analysis of the macular layers, we calculated the mean thickness of the superior, temporal, and inferior sectors of the inner ring of the Early Treatment Diabetic Retinopathy Study-grid for each layer and each patient. This resulted in a single value per layer per patient. For the measurement of the peripapillary retinal nerve fiber layer (pRNFL) thickness an OCT section along a ring of $3.6 \mathrm{~mm}$ diameter centered on the disc was used. pRNFL values were automatically provided by standard Spectralis software and yielded values from 6 sectors around the disc: superotemporal, superonasal, inferotemporal, inferonasal, temporal, and nasal. For this analysis, too, the mean of the superotemporal, the temporal and the inferotemporal sector of the pRNFL was calculated for each patient to simplify statistical analysis. If only 1 eye fulfilled the diagnostic criteria for glaucoma, we used the contralateral eye as control, provided the pRNFL was within normal limits of the Heidelberg system. Given that an apparently normal contralateral eye of a glaucoma patient may not be considered as "healthy" we refer to this group as "normalRNFL" group. To enlarge the normal-RNFL group we additionally included 9 eyes from 9 healthy age-matched subjects that were found in our database. This resulted in 3 groups, glaucoma without MME, glaucoma with MME, and normal-RNFL eyes. For quantitative analysis we excluded the left eye of those patients that had data for

\begin{tabular}{|c|c|c|c|c|c|c|c|c|c|}
\hline Patient ID & Age (y) & Sex & Eye & Diagnosis & BCVA (logMAR) & MD (dB) & pRNFL $(\mu \mathrm{m})$ & mGCL/IPL $(\mu \mathrm{m})$ & mINL/OPL $(\mu \mathrm{m})$ \\
\hline 25 & 81 & $\mathrm{~F}$ & OS & POAG & 0.0 & 6.8 & 59 & 69 & 66 \\
\hline 72 & 68 & M & OD & POAG & 0.1 & 17.9 & 50 & 49 & 86 \\
\hline 81 & 60 & $\mathrm{M}$ & OS & POAG & 0.2 & 19.6 & 34 & 38 & 87 \\
\hline 92 & 56 & M & OD & POAG & 0.2 & & 51 & 58 & 86 \\
\hline 100 & 48 & $\mathrm{~F}$ & OD & POAG & 0.05 & 20.3 & 56 & 64 & 79 \\
\hline 198 & 75 & $\mathrm{~F}$ & OS & PEXG & 0.0 & 15.5 & 59 & 43 & 75 \\
\hline 203 & 72 & $\mathrm{~F}$ & $\mathrm{OD}$ & PEXG & 0.3 & 19.2 & 41 & 43 & 88 \\
\hline 212 & 67 & M & OD & PEXG & 0.0 & 11.8 & 52 & 46 & 83 \\
\hline
\end{tabular}

BCVA indicates best-corrected visual acuity; F, female; M, male; MD, mean visual field defect; $\mathrm{mGCL} / \mathrm{IPL}$, macular ganglion cell layer/inner nuclear layer; $\mathrm{mINL} / \mathrm{OPL}$, macular inner nuclear layer/outer plexiform layer; MME, microcystic macular edema; OD, right eye; OS, left eye; PEXG, pseudoexfoliation glaucoma; POAG, primary open-angle glaucoma; pRNFL, peripapillary retinal nerve fiber layer. 


\begin{tabular}{lcccccc}
\hline \multicolumn{2}{l}{ TABLE 2. Layer Thickness, MD and pRNFL in Normal-RNFL (Ctrl), Glaucoma Patients (Glauc), and Glaucoma Patients With MME } \\
\hline & Ctrl & Glaucoma & MME & Glauc. vs. Ctrl & MME vs. Ctrl & MME vs. Glauc. \\
\hline Eyes (patients) & 25 & 210 & 8 & Sig. & Sig. & Sig. \\
Age & $69 \pm 2$ & $72 \pm 0.8$ & $66 \pm 3.7$ & 0.2 & 0.5 & $<.2$ \\
MD & $1.2 \pm 0.3$ & $6.2 \pm 0.5$ & $16 \pm 1.9$ & 0.001 & $<0.001$ & $<0.001$ \\
pRNFL & $117 \pm 2.5$ & $80 \pm 1.5$ & $50 \pm 3.1$ & $<0.001$ & $<0.001$ & $<0.001$ \\
mGCL/IPL & $91 \pm 1.3$ & $73 \pm 1.0$ & $51 \pm 4.0$ & $<0.001$ & $<0.001$ \\
mINL/OPL & $68 \pm 0.9$ & $68 \pm 0.3$ & $81 \pm 2.6$ & 0.9 & 0.03 & 0.001 \\
mORT & $152 \pm 1.9$ & $154 \pm 0.6$ & $161 \pm 3.0$ & 0.2 & $<$ & 0.04 \\
\hline
\end{tabular}

Mean values are reported with SE of the mean. Mean values were compared between the 3 groups and significance levels (sig.) are reported for each comparison.

MD indicates mean defects; mGCL/IPL, macular ganglion cell layer/inner nuclear layer; mINL/OPL, macular inner nuclear layer thickness and outer plexiform layer; MME, microcystic macular edema; mORT, macular outer retinal thickness; pRNFL, peripapillary retinal nerve fiber layer.
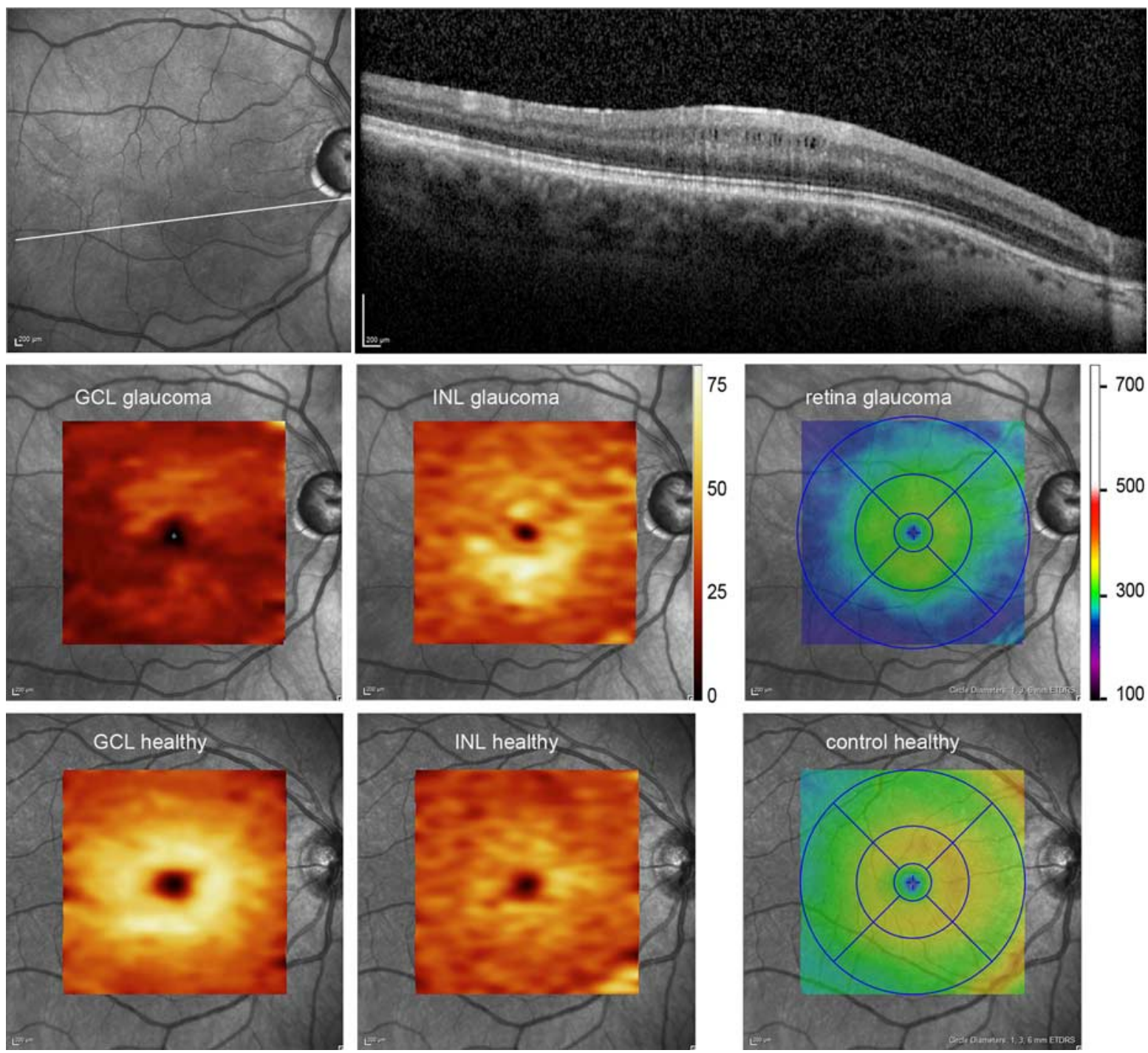

FIGURE 1. Anatomic characteristics of microcystic macular edema in a glaucoma patient. The infrared image of the right eye is shown. The location of the optical coherence tomography (OCT) section is indicated with a white line. The OCT section shows vertical vacuolar inclusions in the inner nuclear layer. Ganglion cell layer (GCL) thickness map of that patient shows marked ganglion cell loss that respects the temporal raphe thus consistent with a nerve fiber bundle loss. Thickness map of the inner nuclear layer, shows macular inner nuclear layer thickness and outer plexiform layer thickening in areas that are most affected by ganglion cell loss. The corresponding thickness maps of a normal-retinal nerve fiber layer eye without apparent glaucoma are shown to allow visual comparison. Figure 1 can be viewed in color online at www.glaucomajournal.com. 

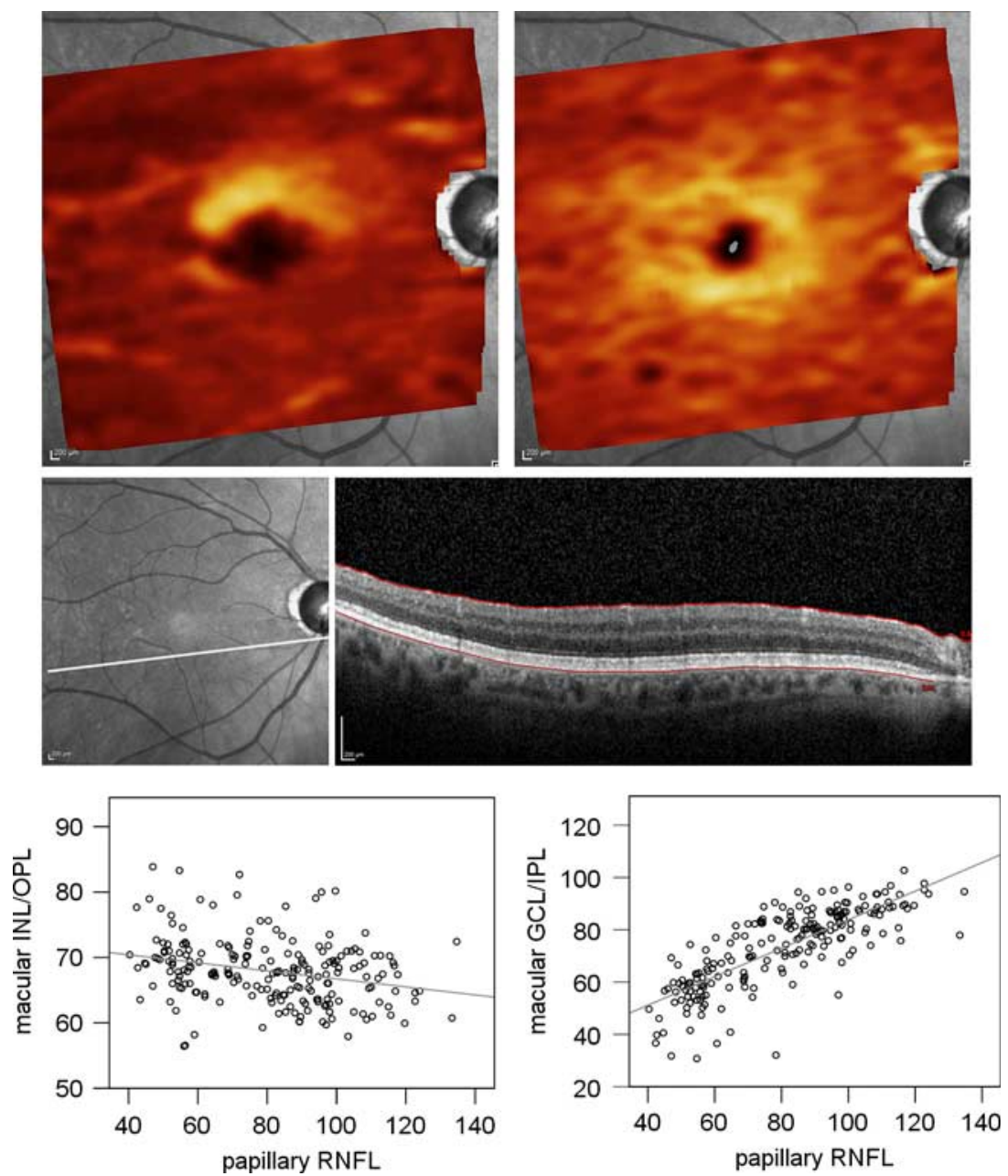

FIGURE 2. Macular optical coherence tomography section of a patient with advanced glaucoma but without microcystic macular edema. Ganglion cell layer (GCL) thickness map (top left) shows severe atrophy in the perifoveal GCL. The corresponding inner nuclear layer (INR) thickness map (top right) shows a thickening, indicating presence of retrograde maculopathy in the absence of microcystic macular edema. Scatter plots of peripapillary retinal nerve fiber layer (RNFL) and macular GCL/inner plexiform layer (IPL) show an excellent correlation of these 2 parameters. Scatter plot of pRNFL and macular inner nuclear layer thickness and outer plexiform layer (mINL/OPL) thickness of all patients shows a significant inverse correlation of $\mathrm{mINL} / \mathrm{OPL}$ thickness with glaucoma severity, thus predicting cases of retrograde maculopathy without microcystic macular edema. Figure 2 can be viewed in color online at www. glaucomajournal.com.

both eyes, such that only 1 eye per patient was included in the statistical analysis. Mean values were compared with analysis of variance. Correlation of parameters were computed with the Pearson test (IBM, SPSS Statistics, Version $21)$. Values are reported as mean $\pm \mathrm{SE}$ of the mean.

\section{RESULTS}

We identified 282 eyes from 218 patients with confirmed high-tension glaucoma and good quality macular OCT. One hundred fifty-nine eyes $(56 \%)$ were from patients with POAG and 123 eyes (44\%) were from patients with PEXG. From 282 glaucoma eyes a total of 8 eyes $(2.8 \%)$ from 8 patients $(3.7 \%)$ showed MME, 3 eyes were from the PEXG group and 5 eyes were from the POAG group (Table 1). The normal-RNFL group was composed of 16 contralateral eyes of patients that did not fulfill the diagnostic criteria for glaucoma and 9 eyes from
9 healthy nonrelated subjects. This normal-RNFL group was comparable in age to the glaucoma patients $(P=0.18)$. No MME was found in the normal-RNFL group.

For quantitative and statistical analysis only a single eye per patient was used to prevent bias. This left us with 98 eyes with PEXG and 112 eyes with POAG. In our cohort patients with PEXG were significantly older than POAG patients $(77 \pm 0.8$ and $68 \pm 1.2 \mathrm{y}$ for $\mathrm{PEXG}$ and $\mathrm{POAG}$ patients, respectively, $P<0.001)$. $\mathrm{mINL} / \mathrm{OPL}$ was about $3 \mu \mathrm{m}$ thinner in PEXG patients as compared with POAG patients (INL/OPL thickness in PEXG patients $=66 \pm$ $0.4 \mu \mathrm{m}, \mathrm{mINL} / \mathrm{OPL}$ thickness in POAG patients $=69 \pm$ $0.5 \mu \mathrm{m}, P=0.001)$. All other parameters, that is $\mathrm{MD}$ in visual field, $\mathrm{mGCL}$ (mGCL/IPL), mINL/OPL, mORT, and temporal pRNFL thickness were not statistically different between the 2 glaucoma groups ( $P>0.35$ for all). Owing to missing data the sample size of mean visual field defect was 


\begin{tabular}{|c|c|c|c|c|c|}
\hline & MD & pRNFL & mGCL/IPL & mINL/OPL & mORT \\
\hline \multicolumn{6}{|l|}{ MD } \\
\hline Corr.coeff. & & $-0.622 \dagger$ & $-0.705 \dagger$ & $0.187^{*}$ & 0.029 \\
\hline Sig. & & 0.000 & 0.000 & 0.016 & 0.715 \\
\hline $\mathrm{n}$ & & 166 & 166 & 166 & 166 \\
\hline \multicolumn{6}{|l|}{ pRNFL } \\
\hline Corr.coeff. & $-0.622 \dagger$ & & $0.791 \dagger$ & $-0.272 \dagger$ & -0.062 \\
\hline Sig. & 0.000 & & 0.000 & 0.000 & 0.372 \\
\hline $\mathrm{n}$ & 166 & & 210 & 210 & 210 \\
\hline \multicolumn{6}{|l|}{$\mathrm{mGCL} / \mathrm{IPL}$} \\
\hline Corr.coeff. & $-0.705 \dagger$ & $0.791 \dagger$ & & $-0.158^{*}$ & -0.011 \\
\hline Sig. & 0.000 & 0.000 & & 0.022 & 0.879 \\
\hline $\mathrm{n}$ & 166 & 210 & & 210 & 210 \\
\hline \multicolumn{6}{|l|}{ mINL/OPL } \\
\hline Corr.coeff. & $0.187^{*}$ & $-0.272 \dagger$ & $-0.158^{*}$ & & 0.023 \\
\hline Sig. & 0.016 & 0.000 & 0.022 & & 0.739 \\
\hline $\mathrm{n}$ & 166 & 210 & 210 & & 210 \\
\hline \multicolumn{6}{|l|}{ mORT } \\
\hline Corr.coeff. & 0.029 & -0.062 & -0.011 & 0.023 & \\
\hline Sig. & 0.715 & 0.372 & 0.879 & 0.739 & \\
\hline $\mathrm{n}$ & 166 & 210 & 210 & 210 & \\
\hline
\end{tabular}

Pearson correlation coefficients (corr.coeff.), statistical significance (sig.), and number of eyes (n) are reported. Except for outer retinal thickness all parameters show correlation with each other. Patients with MME were excluded for this analysis.

*Correlation is significant at the 0.05 level.

†Correlation is significant at the 0.01 level.

MD indicates mean defects; mGCL/IPL, macular ganglion cell layer/inner nuclear layer; mINL/OPL, macular inner nuclear layer thickness and outer plexiform layer; MME, microcystic macular edema; mORT, macular outer retinal thickness; pRNFL, peripapillary retinal nerve fiber layer.

$\mathrm{n}=166$ instead of 210 , which reduced statistical power for this parameter. We combined the PEXG group and the POAG group into a single glaucoma group for the remainder of the analysis. Compared with the non-MME glaucoma group the patients with MME had more advanced glaucoma with more visual field defect, reduced pRNFL, and reduced mGCL/IPL thickness $(P<0.001$ for each comparison, Table 2). We found a significantly thicker mINL/OPL in MME patients compared with the glaucoma group or normal RNFLs $(P<0.001)$. The vertical microcysts were exclusively present in the inner nuclear layer and were located in a perifoveal ring sparing the fovea itself (Fig. 1).

We next investigated the deeper retinal layers in those patients that did not show MME. As MME patients show obvious involvement of deeper retinal layers, eyes with MME were excluded from the following analysis. We found that our glaucoma group had a significant visual field loss (MD in glaucoma group $=6.2 \pm 0.5$, MD in normalRNFL group $=1.2 \pm 0.3, P<0.001)$. This field loss was associated with a thinning of the papillary nerve fiber layer ( $\mathrm{pRNFL}=80 \pm 1.5$ and $117 \pm 2.5 \mu \mathrm{m}$ in glaucoma and normal-RNFL patients, respectively) and with a thinning of the $\mathrm{mGCL}(\mathrm{mGCL} / \mathrm{IPL}=73 \pm 1 \mu \mathrm{m}$ in glaucoma and $91 \pm 1 \mu \mathrm{m}$ in normal RNFL, $P<0.001)$. Of note, we found no difference of the mean mINL/OPL thickness and mORT between glaucoma patients and the normal-RNFL group $(P \geq 0.2$ for both). Thus, average retinal layers beneath GCL are not different between the normal-RNFL group and the glaucoma group. Next we tested whether there is a correlation of glaucoma severity and individual retinal layers. We found a strong inverse correlation of visual field defect with both $\mathrm{pRNFL}$ and $\mathrm{mGCL} / \mathrm{IPL}$ thickness $(P<0.001$ for both, Fig. 2 and Table 3$)$. A positive correlation was found between the visual field defect and mINL/OPL $(P=0.016)$. Similarly we found a significant correlation of pRNFL with mGCL/IPL $(P<0.001)$ and an inverse correlation with $\mathrm{mINL} / \mathrm{OPL}(P<0.001$, Table 3 and Fig. 2). No significant correlation was found for the deeper retinal layers $(P=0.37$, Table 3$)$.

\section{DISCUSSION}

In this study we found that glaucoma-associated macular layer changes are not limited to the ganglion cell level: First, we found MME in about 3\% of glaucoma eyes. Second, we found a significant correlation of mINL/ OPL thickness with glaucoma severity, that is an inverse correlation of $\mathrm{mGCL} / \mathrm{IPL}$ thickness and mINL/OPL thickness. This correlation may explain why only some cases of optic neuropathy develop MME: ganglion cell loss may lead to a gradual thickening of mINL/OPL, which in advanced cases may result in clinically visible MME. Consistent with this hypothesis we found that glaucoma was significantly worse in patients with MME as compared with the remaining glaucoma group. The correlation with severity may also help to understand the variable MME prevalence previously reported in different types of optic neuropathies. Some optic neuropathies, including neuromyelitis optica, cause more severe optic atrophy and have a prevalence of MME up to $30 \%,{ }^{14,22}$ while more moderate optic neuropathies, such as those in MS, have a lower prevalence of about $4 \% .{ }^{23}$ We found a frequency of MME of $3 \%$ in the glaucoma group, while we previously found a prevalence of $8 \%$ in nonglaucomaassociated optic neuropathy. ${ }^{13}$ Hasegawa et $\mathrm{al}^{11}$ found a higher MME frequency of $6 \%$ in POAG eyes. The latter difference in frequency may be explained by the higher 
number of advanced glaucoma cases in their cohort compared with our study. Other factors not within the scope of this study may also contribute to the formation of MME. Youth appeared to be a risk factor for $\mathrm{MME}$ in our previous study of nonglaucomaassociated optic neuropathy. ${ }^{13}$ Indeed the average age of the glaucoma group in this study was approximately 70 , as compared with $<50$ in our previous study. ${ }^{13}$ Consistent with age as a possible risk factor, we found that glaucoma patients with MME were on average 6 years younger than the rest of the glaucoma group. However, the small MME group size prevents statistical analysis and the age difference may be coincidental. The correlation of inner nuclear layer thickness with glaucoma severity raises the possibility that mINL/OPL can be thickened without apparent MME (as seen in Fig. 2). We thus suggest the term "retrograde maculopathy" to describe optic neuropathy-associated macular changes, only some of which may present with MME. The presence of retrograde maculopathy with MME and alterations in the deeper retinal layer raises the questions whether these changes are caused by glaucoma or its associated treatments, and how specific to glaucoma these changes actually are. A series of previous publications showed that optic neuropathy of any origin may be associated with MME. ${ }^{13,24,25}$ We thus presume that retrograde maculopathy is a nonspecific finding of any optic neuropathy and the main causal link to glaucoma is that this disease also leads to an optic neuropathy. However, this does not exclude the possibility that some glaucoma-specific factors, such as medication, may also play a role in the development of retrograde maculopathy.

In accordance with Leung et $\mathrm{al}^{5}$ and Wang et al, ${ }^{9}$ no difference of $\mathrm{mINL} / \mathrm{OPL}$ thickness between normalRNFLs and glaucoma patients was found. This seems contradictory to our finding of an inverse correlation of $\mathrm{mGCL} / \mathrm{IPL}$ thickness and mINL/OPL thickness. There are 3 possibilities to explain this: (1) The normal-RNFL group may not be valid. One limitation of our study is the small normal-RNFL group, which mixes eyes from healthy patients and eyes of patients that are not clinically affected by glaucoma. Thus the comparisons with the normal-RNFL group must be interpreted with caution; (2) the increased thickness may not be detectable in the entire group due to lack of statistical power; (3) a more likely hypothesis is that optic neuropathy is associated with some atrophy in the inner nuclear layer. This atrophy leads to an overall thickness reduction which is counterbalanced by increased water content and thus is associated with concurrent atrophy and swelling. The latter may be predominant in advanced cases. Postmortem analysis from patients with long standing optic neuropathy indeed found a reduced cell count in the inner nuclear layer. ${ }^{26}$

On the basis of our finding that GCL thinning can be associated with $\mathrm{mINL} / \mathrm{OPL}$ thickening, we suggest to refrain from relying on total macular thickness as a parameter for glaucoma progression, but rather measure the GCL alone.

In summary, our data show that glaucomatous damage can be detected at the level of macular ganglion cells and may also affect the deeper retinal layers in the perimacular area. We suggest that the term retrograde maculopathy in glaucoma suitably describes the structural findings of thinning of the GCL, thickening of the underlying inner nuclear and outer plexiform layers, and/or MME in advanced glaucoma cases.

\section{REFERENCES}

1. Wollstein G, Schuman JS, Price LL, et al. Optical coherence tomography longitudinal evaluation of retinal nerve fiber layer thickness in glaucoma. Arch Ophthalmol. 2005;123: 464-470.

2. Leung CK-S. Diagnosing glaucoma progression with optical coherence tomography. Curr Opin Ophthalmol. 2014;25: 104-111.

3. Leung CK-S, Cheung CY-L, Weinreb RN, et al. Retinal nerve fiber layer imaging with spectral-domain optical coherence tomography: a variability and diagnostic performance study. Ophthalmology. 2009;116:1257-1263. 1263.e1-2.

4. Curcio CA, Allen KA. Topography of ganglion cells in human retina. J Comp Neurol. 1990;300:5-25.

5. Leung CKS, Ye C, Weinreb RN, et al. Impact of age-related change of retinal nerve fiber layer and macular thicknesses on evaluation of glaucoma progression. Ophthalmology. 2013;120: 2485-2492.

6. Na JH, Sung KR, Baek S, et al. Detection of glaucoma progression by assessment of segmented macular thickness data obtained using spectral domain optical coherence tomography. Invest Ophthalmol Vis Sci. 2012;53:3817-3826.

7. Choi SS, Zawadzki RJ, Lim MC, et al. Evidence of outer retinal changes in glaucoma patients as revealed by ultra highresolution in vivo retinal imaging. Br J Ophthalmol. 2011;95: $131-141$.

8. Werner JS, Keltner JL, Zawadzki RJ, et al. Outer retinal abnormalities associated with inner retinal pathology in nonglaucomatous and glaucomatous optic neuropathies. Eye (Lond). 2011;25:279-289.

9. Wang M, Hood DC, Cho J-S, et al. Measurement of local retinal ganglion cell layer thickness in patients with glaucoma using frequency-domain optical coherence tomography. Arch Ophthalmol. 2009;127:875-881.

10. Tan O, Chopra V, AT-H Lu, et al. Detection of macular ganglion cell loss in glaucoma by Fourier-domain optical coherence tomography. Ophthalmology. 2009;116:2305-2314.

11. Hasegawa T, Akagi T, Yoshikawa M, et al. Microcystic inner nuclear layer changes and retinal nerve fiber layer defects in eyes with glaucoma. PLoS One. 2015;10:e130175.

12. Balk LJ, Killestein J, Polman CH, et al. Microcystic macular oedema confirmed, but not specific for multiple sclerosis. Brain. 2012;135(pt 12):e226. Author reply e227.

13. Abegg M, Dysli M, Wolf S, et al. Microcystic macular edema: retrograde maculopathy caused by optic neuropathy. Ophthalmology. 2014;121:142-149.

14. Sotirchos ES, Saidha S, Byraiah G, et al. In vivo identification of morphologic retinal abnormalities in neuromyelitis optica. Neurology. 2013;80:1406-1414.

15. Mahroo OA, Shalchi Z, Kisimbi J, et al. Re: Abegg et al: Microcystic macular edema: retrograde maculopathy caused by optic neuropathy (Ophthalmology 2014;121:142-9). Ophthalmology. 2014;121:e40.

16. Scherer J, Schnitzer J. Intraorbital transection of the rabbit optic nerve: consequences for ganglion cells and neuroglia in the retina. J Comp Neurol. 1991;312:175-192.

17. Huxlin KR, Dreher Z, Schulz M, et al. Glial reactivity in the retina of adult rats. Glia. 1995;15:105-118.

18. Hiscott PS, Grierson I, Trombetta CJ, et al. Retinal and epiretinal glia - an immunohistochemical study. $\mathrm{Br} J$ Ophthalmol. 1984;68:698-707.

19. Wen JC, Freedman SF, El-Dairi MA, et al. Microcystic macular changes in primary open-angle glaucoma. J Glaucoma. 2016;25:258-262.

20. Schulze-Bonsel K, Feltgen N, Burau H, et al. Visual acuities "hand motion" and "counting fingers" can be quantified with the Freiburg visual acuity test. Invest Ophthalmol Vis Sci. 2006; $47: 1236-1240$. 
21. Dufour PA, Ceklic L, Abdillahi H, et al. Graph-based multisurface segmentation of OCT data using trained hard and soft constraints. IEEE Trans Med Imaging. 2013;32:531-543.

22. Gelfand JM, Cree BA, Nolan R, et al. Microcystic inner nuclear layer abnormalities and neuromyelitis optica. JAMA Neurol. 2013;70:629-633.

23. Gelfand JM, Nolan R, Schwartz DM, et al. Microcystic macular oedema in multiple sclerosis is associated with disease severity. Brain. 2012;135(pt 6):1786-1793.
24. Abegg M, Zinkernagel M, Wolf S. Microcystic macular degeneration from optic neuropathy. Brain. 2012;135(pt 12):e225.

25. Burggraaff MC, Trieu J, de Vries-Knoppert WAEJ, et al. The clinical spectrum of microcystic macular edema. Invest Ophthalmol Vis Sci. 2014;55:952-961.

26. Gills JP, Wadsworth JA. Degeneration of the inner nuclear layer of the retina following lesions of the optic nerve. Trans Am Ophthalmol Soc. 1966;64:66-88. 